\title{
SINGLE-CENTRE EXPERIENCE WITH MECHANOCHEMICAL ABLATION OF INSUFFICIENT VEINS WITH THE FLEBOGRIF® CATHETER IN A 36-MONTH FOLLOW-UP
}

Marek Itżecki, Piotr Terlecki, Stanisław Przywara, Tomasz Zubilewicz

Department of Vascular Surgery and Angiology, Medical University of Lublin, Lublin, Poland

\begin{abstract}
Introduction: Flebogrif is the youngest representative of the MOCA treatment group. Its minimally invasive nature and high effectiveness contribute to its popularity. In the presented study, its effectiveness was assessed over a 36-month follow-up. Material and methods: Observations were conducted over a 36-month period as a prospective study. Two hundred patients meeting the established inclusion criteria were enrolled in the study, including 170 women aged 18 to 87 , mean age 56 years, and 30 men, age 23 to 75 , mean age 46 years. The obtained numerical data were statistically analysed using tests for variables with nonparametric distribution: Wilcoxon test and Friedman's ANOVA. The follow-up visits were scheduled as follows: baseline, 1 , and 4 weeks and 3, 6, 12, 24, and 36 months. Numerical data were collected based on the assessment validation of venous clinical severity score (VCSS) and visual analogue scale (VAS). Ultrasound evaluation was performed to assess the anatomical effectiveness of the procedure. The safety of the method was assessed in terms of the severity and number of complications.

Results: Based on the VCSS and VAS scale assessment, a statistically significant decrease in the intensity of clinical symptoms was observed both between monitoring visits and in relation to the baseline. One case of deep vein thrombosis $(0.5 \%), 1$ prolongated pain, 35 thrombophlebitis, and 28 discolourations (32\%) were reported. The 12 -month success rate dropped to $92 \%$ and remained there until 36 months.

Conclusions: A high success rate of $92 \%$ was achieved, which is comparable with other methods currently used in the ablation of insufficient superficial veins. High safety of the method and low number of complications is expressed. Good cosmetic effect. A quick return to previous physical activity is an additional incentive for its use.
\end{abstract}

Key words: varicose veins, MOCA, Flebogrif

\section{ORIGINAL PAPER}

Phlebological Review 2021; 29, 1:32-37

DOl: https://doi.org/10.5114/pr.2021.106880

Submitted: 26.05 .2021

Accepted: 31.05 .2021

ADDRESS FOR CORRESPONDENCE

Prof. Marek Itzecki

Chair and Department

of Vascular Surgery and Angiology

Medical University of Lublin, Poland

e-mail: ilzecki.m@gmail.com

\section{INTRODUCTION}

Recent years have brought a number of solutions for the treatment of venous insufficiency. It seems that thermal techniques such as EVLT, RFA, and STEAM dominate the medical market thanks to the effectiveness and repeatability of the therapeutic effect [1]. The ClariVein method proposed by Michael Toll was a new opening in the field of minimally invasive procedures for the treatment of superficial venous insufficiency [2]. The essence of the procedure was a combination of 2 factors: mechanical damage combined with chemical destruction of the endothelium. The immediate effect of the procedure was the closure of the vein lumen by a formed hemoclot, while the initiated chronic inflammation led to its fibrosis and permanent occlusion [3]. Follow-up over a period of 12 months showed an effectiveness of $88-100 \%$ [4].

In 2011, Professor Piotr Ciosek, Head of the First Chair and Clinic of General and Vascular Surgery, II Fac- ulty of Medicine, Medical University of Warsaw, presented an idea for a device whose essence of operation referred to the concept of mechanical-chemical ablation [5]. An innovative solution was to replace the rotating core with a ball, as is the case with the ClariVein ${ }^{\circ}$ catheter (which causes several unexpected, unpleasant complications), with 5 cutting elements damaging the endothelial surface (Fig. 1) [6]. The currently used catheter is the result of a number of technological and functional improvements patented by Balton Sp. z o. o., increasing its effectiveness and, above all, safety. The many advantages of the new Flebogrif ${ }^{\circ}$ catheter include its simplicity, intuitive operation, and durability. A unique feature is its ability to adapt the radial force of the cutting elements to the diameter of the vein, which increases the effect of the mechanical MOCA phase with the use of a Flebogrif. aAnalysis of histological images confirmed the effectiveness of the working part (cutting elements) of the catheter. Dam- 
age to the endothelial surface caused by the action of the hooks penetrated the muscular layer of the vessel wall.

\section{MATERIAL AND METHODS}

The effectiveness of the method using the Flebogrif ${ }^{\circledR}$ catheter was analysed in relation to other treatments. In our own material, 200 patients were subjected to ablation of the insufficient vein of the great saphenous vein/small saphenous vein system. The authors obtained approval for the study based on the decision of the Bioethics Committee (KF-0254/226/2015). Patients presented features of venous insufficiency classified according to the CEAP scale between $\mathrm{C}_{2}$ and $\mathrm{C}_{6}$. Table 1 presents the demographic data and clinical cardiovascular disease advancement of the study group.

In the adopted study plan, follow-up visits were arranged in the following order: baseline, 1 , and 4 weeks and $1,3,6,12,24$, and 36 months. The intensity of clinical symptoms of the disease was classified using the venous clinical severity score (VCSS) scale, ranging between 7 and 21 points. During the established observation period, a slow decline in the study participants was noted, which to some extent explains the radical improvement after the surgery. A quick return to full physical activity and a significant reduction in the symptoms associated with the underlying disease contributed to the resignation of a certain

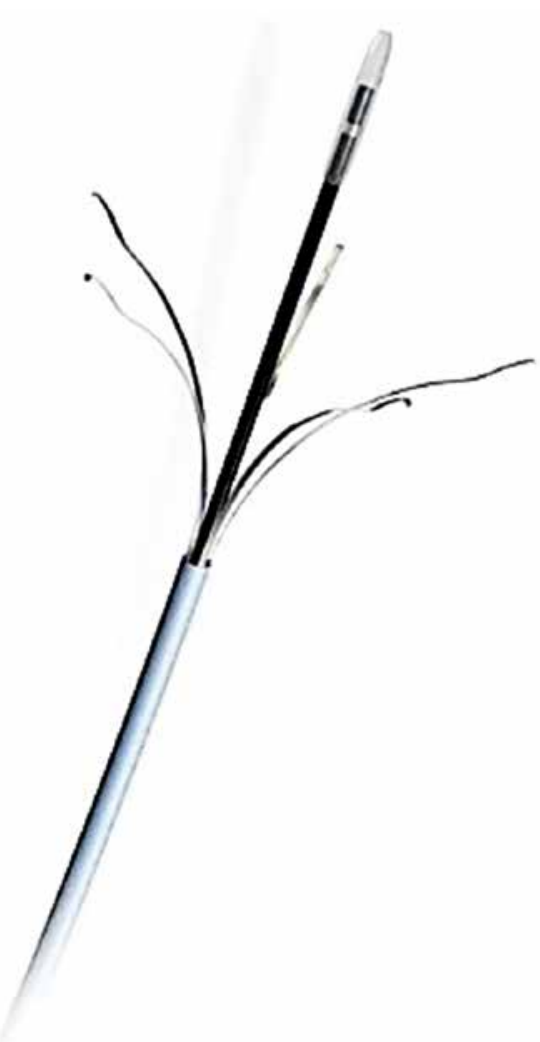

Fig. 1. Flebogrif ${ }^{\circledast}$ catheter

Table 1. Demographic data of the observed group (age, gender), numerical distribution of the study group according to the CEAP scale, and the age range of the operated patients

\begin{tabular}{|c|c|c|c|c|c|c|c|}
\hline \multirow[t]{3}{*}{ Scale } & \multicolumn{6}{|c|}{ Age } & \multirow[t]{3}{*}{$p$} \\
\hline & \multicolumn{3}{|c|}{$\leqslant 56.5$} & \multicolumn{3}{|c|}{$>56.5$} & \\
\hline & $n$ & $M$ & SD & $n$ & $M$ & SD & \\
\hline VCSS-b & 100 & 9.40 & 3.76 & 100 & 12.03 & 3.74 & $<0.001$ \\
\hline VCSS-1 & 87 & 6.99 & 3.86 & 95 & 9.08 & 3.65 & $<0.001$ \\
\hline VCSS-3 & 85 & 4.93 & 3.43 & 94 & 6.57 & 3.44 & $<0.001$ \\
\hline VCSS-6 & 83 & 3.83 & 3.15 & 91 & 5.58 & 2.88 & $<0.001$ \\
\hline VCSS-12 & 77 & 3.64 & 2.98 & 91 & 5.05 & 2.75 & $<0.001$ \\
\hline VCSS-24 & 67 & 4.18 & 2.92 & 91 & 5.04 & 2.56 & 0.0930 \\
\hline VCSS-36 & 65 & 4.90 & 2.77 & 89 & 5.17 & 2.22 & 0.8899 \\
\hline VAS-b & 100 & 2.68 & 1.61 & 100 & 3.77 & 1.72 & $<0.001$ \\
\hline VAS-1 & 87 & 1.54 & 1.34 & 95 & 2.20 & 1.44 & $<0.001$ \\
\hline VAS-3 & 85 & 0.62 & 0.98 & 94 & 1.19 & 1.36 & $<0.01$ \\
\hline VAS-6 & 83 & 0.45 & 0.83 & 91 & 0.98 & 1.18 & $<0.001$ \\
\hline VAS-12 & 77 & 0.38 & 0.67 & 91 & 0.78 & 0.96 & $<0.01$ \\
\hline VAS-24 & 67 & 0.29 & 0.51 & 91 & 0.88 & 1.01 & $<0.01$ \\
\hline VAS-36 & 65 & 0.45 & 0.63 & 89 & 0.81 & 0.89 & 0.0772 \\
\hline
\end{tabular}

VCSS - venous clinical severity score, VAS - visual analogue scale 


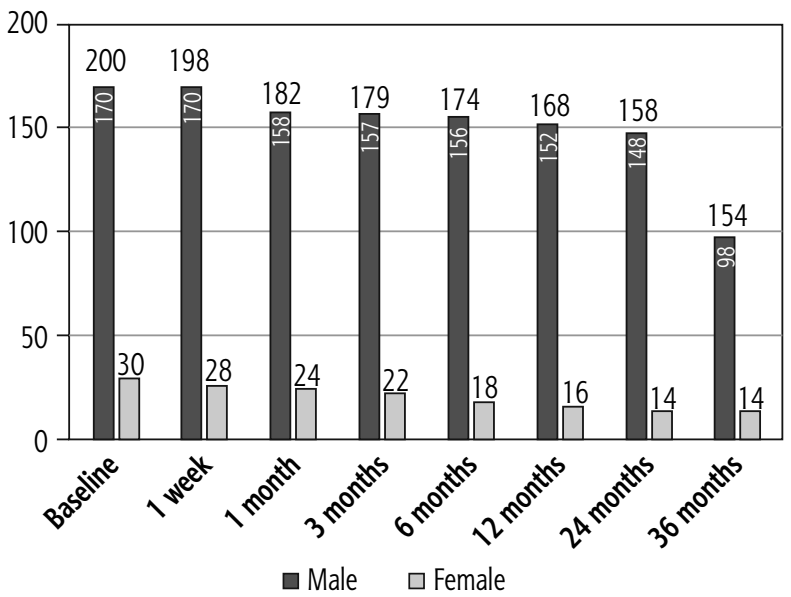

Fig. 2. Number of patients participating in visits during the 36-month follow-up

number of patients from participation in monitoring visits. The interchangeability of the number of patients participating in monitoring visits is presented in Figure 2.

The observed natural decrease in the number of participants in the Flebogrif ${ }^{\infty}$ trial is essential for the full evaluation of the method's effectiveness and is probably the weak point of each clinical trial.

The ablated saphenous vein was punctured at different levels depending on the length of the inefficient segment; in the case of the small saphenous vein it was always punctured at the lowest point of the axial reflux. The numerical distribution of the puncture level is presented in Table 2.

In 172 cases, the saphenous vein was ablated, which accounted for $86 \%$ of the study group, and the small saphenous vein was closed in 28 cases, which accounted for $14 \%$ of the studied group of patients. The range of diameters of the veins treated with the Flebogrif ${ }^{\circ}$ catheter, depending on the side of the operated limb and sex, are presented in Table 3.
The results of our own research were analysed in 4 categories:

- clinical success, defined as an objective improvement in the clinical condition, based on the assessment according to the VCSS scale and the visual analogue scale (VAS),

- effectiveness of the method, expressed as a percentage of closed veins, based on the ultrasound assessment,

- method safety, expressed in terms of observed complications,

- advantages of the catheter/Flebogrif method ${ }^{\circledR}$ including technical.

\section{STATISTICAL ANALYSIS METHODS}

The numerical data obtained during the observation, obtained on the basis of the assessment of clinical symptoms related to the underlying disease using the VCSS (Venous clinical severity score) scale, and the VAS, were statistically analysed using the Wilcoxon tests (for paired samples) and Friedman's ANOVA for the independent variables with nonparametric distribution. In the performed statistical analysis, the behaviour of the observed variable between the established time points (follow-up visits) as well as in relation to the baseline day was assessed. The obtained results showed a statistically significant difference in the intensity of the examined feature as assessed by the VCSS scale between individual visits, and also in relation to day zero (baseline).

\section{RESULTS}

On the basis of the performed statistical analysis, a statistically significant decrease in the intensity of clinical symptoms measured with the VCSS scale and the intensity of pain was found. Among the analysed factors, the age of the patients was most influenced by the differences in the numerical values obtained on the basis of the patient's clinical assessment according to the VCSS scale

Table 2. Numerical distribution of puncture sites of the saphenous vein in females and males

\begin{tabular}{lcccc}
\hline \multirow{2}{*}{ Gender } & \multicolumn{2}{c}{ Puncture site } & \multicolumn{2}{c}{ Below the knee } \\
\cline { 2 - 5 } & Above the knee & Knee level & GSV & SSV \\
\hline Female & 37 & 36 & 72 & 25 \\
\hline Male & 7 & 6 & 15 & 3 \\
\hline
\end{tabular}

GSV - great saphenous vein, SSV - small saphenous vein

Table 3. The range of the vein diameter depends on the puncture level, the side of the operated vein, and gender

\begin{tabular}{cccccccc}
\hline \multicolumn{2}{c}{ Diameter of the vein (from - to/ average) } & \multicolumn{3}{c}{ Operated limb } \\
\hline \multirow{2}{*}{ Below SFJ } & Mid-thigh & Knee level & \multicolumn{3}{c}{ Females } & & Males \\
\cline { 3 - 8 } & & & Right & Left & Right & Left \\
\hline $4.2-22.1 / 8.1$ & $3.8-17.1 / 6.2$ & $3.8-11.9 / 6.6$ & 68 & 102 & 13 & 17 \\
\hline
\end{tabular}


Table 4. Influence of patients' age on the shaping of differences in numerical values obtained from the venous clinical severity score and visual analogue scale

\begin{tabular}{lcccc}
\hline \multirow{2}{*}{ Recanalization } & \multicolumn{2}{c}{ Female } & \multicolumn{2}{c}{ Male } \\
\cline { 2 - 5 } & GSV & SSV & GSV & SSV \\
\hline Full rec. & 5 & 3 & 2 & - \\
\hline Partial r. & 4 & 1 & - & - \\
\hline
\end{tabular}

GSV - great saphenous vein, SSV - small saphenous vein

and the subjective feeling of pain measured with the VAS scale (Table 4).

The largest statistically significant difference was observed between the baseline value and the 12-month follow-up, while the decrease in the intensity of the examined feature between months 12-24 and 24-36 was not statistically significant. Figure 3 shows the graphical form of statistical significance as assessed by VCSS and VAS.

During the 36-month observation, 15 cases of recanalization were recorded, which constituted $8 \%$ of the studied group. Based on the European Consensus for Sclerotherapy the following recanalization classification criteria were adopted: success - no flow of the vein completely closed by fibrous changes, partial recanalization - reflux $<0.5 \mathrm{~s}$, reduction of the vein diameter, failure - reflux $>0.5 \mathrm{~s}$, full vein patency $[7,8]$. Among the 10 identified cases of complete recanalization (according to the adopted criteria), 8 were related to the saphenous vein and 2 to the small saphenous vein. In the partial recanalization group, 4 cases concerned the saphenous vein, and 1 case concerned the small saphenous vein. Table 5 presents the numerical distribution of recanalization in the observed group of patients, taking gender into account.

Safety analysis of the MOCA method using the Flebogrif " ${ }^{\circ}$ catheter during the 36-month follow-up, it showed one case of deep vein thrombosis (DVT) among serious complications. In the course of further diagnostics, the patient was diagnosed with factor $\mathrm{V}$ Leiden mutation. The patient underwent anticoagulant therapy with NOAC drugs. Among minor complications, there was 1 case of prolonged pain, 35 cases of superficial vein thrombosis, and 28 cases of hyperpigmentation. No adverse effects related to the type of sclerosing agent used were observed. After the procedure, patients were

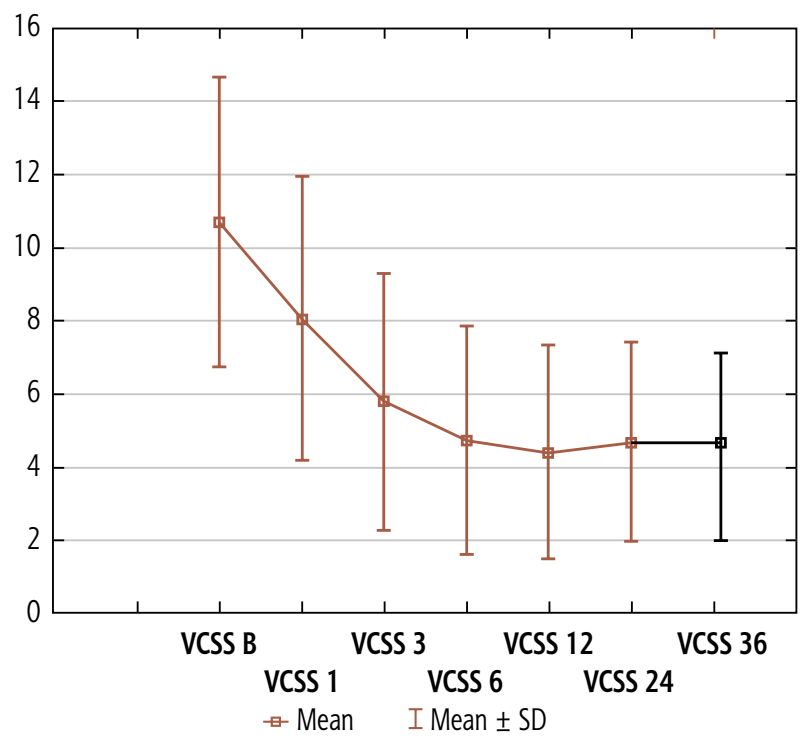

Fig. 3. Variation in the numerical value of VCSS at subsequent follow-up visits over the period of 36 months

discharged home within an hour. The only trace of the procedure was the puncture site.

\section{DISCUSSION}

A method of mechanical-chemical ablation of inefficient veins of the superficial system using the Flebogrif catheter is a valuable supplement to the group of minimally invasive treatments covered by the common name MOCA. The combination of 2 factors of the mechanical destruction of the endothelium and the chemical action of sclerosant significantly increased the effectiveness of the method [9]. Sclerotherapy as an independent procedure used in obliteration of inefficient superficial veins is effective although, according to the literature, it is slightly lower than the currently most commonly used thermal methods, such as EVLT or RFA [10]. It should be emphasized, however, that the relatively low price increases the availability of the procedure, which encourages a certain group of doctors and patients to use it. The undoubted advantage of the Flebogrif, like other treatments (ClariVein), is the elimination of the thermal factor, which significantly widens the possibilities of using the method in areas with a high risk of neurological damage, sometimes accompanying EVLT, RFA, or STEAM [11, 12].

Table 5. Numerical distribution of recanalization/failure by sex and ablated great saphenous vein/small saphenous vein

\begin{tabular}{|c|c|c|c|c|c|c|c|}
\hline $\mathrm{C} 2$ & $92(46 \%)$ & \multirow[t]{2}{*}{ Sex } & \multirow[t]{2}{*}{ Number of patients } & \multicolumn{3}{|c|}{ Age } & \multirow[t]{2}{*}{ Total } \\
\hline C3 & $32(16 \%)$ & & & Max & Average & Min & \\
\hline C4 & $46(23 \%)$ & Females & 170 & 87 & 56 & 18 & \multirow[t]{2}{*}{200} \\
\hline$\frac{C 5}{C 6}$ & $\frac{26(13 \%)}{4(2 \%)}$ & Males & 30 & 75 & 46 & 23 & \\
\hline
\end{tabular}


Unlike Clarivein, in the Flebogrif method $^{\circledR}$ only a foamed sclerosant is used according to the Tessari method in the proportion of 4 volumes of air to 1 volume of Polidocanol $[12,13]$. The obtained results indicate the high efficiency of the method, reaching $92 \%$ of closed veins in a 36-month follow-up. The vast majority of failures were observed in the first 6 months (8 at the 3-month visit and 6 cases at the 6-month visit, respectively), which to some extent should be explained by the learning curve. In the remaining months, isolated cases of recanalization were observed. Another important advantage of the Flebogrif catheter is that there is no need to use tumescence, which is sometimes the cause of haematomas, arterial damage, and trunks of nerves accompanying the vein, which are important complications associated with thermal ablation [14]. A distinguishing feature of the method is the possibility of repeating the mechanical damage to the vessel wall through the cutting part of the Flebogrif catheter, while maintaining the original position of the guide. The undoubted advantage of the Flebogrif catheter, as well as other MOCA methods, is the lack of the need for anaesthesia (general, epidural). An additional advantage of the method is the short duration of the procedure (10-15 minutes). The complete set delivered at the factory allows the procedure to be performed without the need to invest in additional equipment. The very good visibility of the elements of the working part of the catheter in the ultrasound image allows for precise positioning in a dedicated place, i.e. $2-2.5 \mathrm{~cm}$ from the mouth of the saphenous vein to the femoral vein. Another important feature of the catheter/Flebogrif method ${ }^{\circledast}$ is a rapid return of the patient to previous physical activity, which is essential for the younger group of patients. The disadvantage of the method using the Flebogrif catheter is the inability to ablate insufficient perforators, which is determined by the specificity of its construction. The simplicity of its construction and, consequently, its simple and intuitive operation are another positive of the discussed method of mechanical and chemical ablation of veins. During the entire observation period, no mechanical defects were found that could, on the one hand, affect the safety of the procedure, and on the other hand, its effectiveness. Lene and Moore described an interesting complication related to the unintentional wrapping of a vein around the rotating tip of the ClariVein ${ }^{\star}$ working part, which radically changed the original nature of the procedure from minimally invasive to the classic painful saphenectomy [6]. However, these kinds of complications constitute a fraction of a percent.

Contraindications to MOCA using the Flebogrif catheter ${ }^{\oplus}$, as with other endovascular procedures, are as follows: the current DVT and its occlusion/obstruction, pregnancy, acute infectious disease, lower limb ischaemia, severe lymphoedema, bleeding diathesis, allergic reaction to the sclerosant, or local skin infection.
Summing up, it should be stated that the obtained high effectiveness rate of the method using the Flebogrif catheter is a promise of therapeutic repeatability, which seems to be the main argument for further use. It should also be remembered that the apparently natural decrease in the number of patients participating in monitoring visits (typical for most studies) may to some extent affect the actual success/failure rate due to the lack of information on the outcome of surgery in those patients with potential recanalization.

\section{CONCLUSIONS}

A high success rate of up to $92 \%$ was achieved, which is comparable with the results of other methods currently used in the ablation of insufficient superficial veins. The high safety of the method and low number of complications were expressed, as well as good cosmetic effect and a quick return to previous physical activity is an additional incentive to use it.

The authors declare no conflict of interest.

\section{References}

1. Elrasheid A, Kheirelseid H, Gillian C, et al. Systematic review and meta-analysis of randomized controlled trials evaluating long-term outcomes of endovenous management of lower extremity varicose veins. J Vasc Surg Venous Lymphat Disord 2018; 6: 256-270.

2. Elias S, Raines JK. Mechanochemical tumescentless endovenous ablation: final results of a preliminary clinical study. Phlebology 2012; 27: 67-72.

3. Mueller RL, Raines JK. ClariVein mechanochemical ablation: background and procedural details. Vasc Endovascular Surg 2013; 47: 195-206.

4. Witte ME, Zeebregts CJ, de Borst GJ, Reijnen MMPJ, Boersma D. Mechanochemical endovenous ablation of saphenous veins using the ClariVein: a systematic review. Phlebology 2017; 32: 649-657.

5. Ciostek P, Kowalski M, Woźniak W, et al. Phlebogriffe - a new device for mechanical-chemical ablation of insufficient saphenous veins: a pilot study. Phlebological Rev 2015; 23: 72-77.

6. Lane TR, Moore HM, Franklin IJ, Davies AH. Retrograde inversion stripping as a complication of the ClariVein mechanochemical venous ablation procedure. Ann R Coll Surg Engl 2015; 97: e18-20.

7. Breu FX, Guggenbischler S, Wollmann JC. Europäische Konsensuskonferentz zur Schaumaklerotherapie der Varikose. Gefässchir 2011;16: 236-241.

8. Rabe E, Brief FX, Cavezzi A, et al. Guideline group. European guidelines for sclerotherapy in chronic venous disorders. Phlebology 2014; 29: 338-354.

9. Mueller RL, Raines JK. ClariVein mechanochemical ablation: background and procedural details. Vasc Endovascular Surg 2013; 47: 195-206.

10. Van Eekeren RR, Boersma D, de Vries JP, Zeebregts CJ, Reijnen MM. Endovenous treatment of insufficient saphenous vein 
diseases - literature review update. Semin Vasc Surg June 2014; 27: 118-136.

11. Nemoto H, Mo M, Ito T, et al. Japanese Endovenous Ablation Committee for Varicose Veins. Venous thromboembolism complications after endovenous laser ablation for varicose veins and role of duplex ultrasound scan. J Vasc Surg Venous Lymphat Disord 2019; 7: 817-823.

12. Woźniak W, Mlosek RK, Ciostek P. Complications and failure of endovenous laser ablation and radiofrequency ablation procedures in patients with lower extremity varicose veins in a 5-year follow-up. Vasc Endovascular Surg 2016; 50: 475-483.

13. Costantino DC, Antonino S, Fiorillo A, Matula ThJ. Size of sclerosing foams prepared by ultrasound, mechanical agitation, and the handmade tessari method for treatment of varicose veins. J Ultrasound Med 2017; 36: 649-658.

14. Bunke N, Brown K, Bergan J. Foam sclerotherapy: techniques and uses. Perspect Vasc Surg Endovasc Ther 2009; 21: 91-93. 